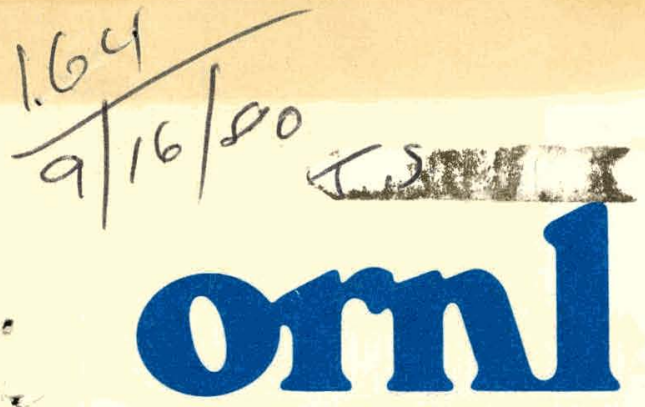

OAK

RIDGE

NATIONAL

LABORATORY

UNION

CARBIDE

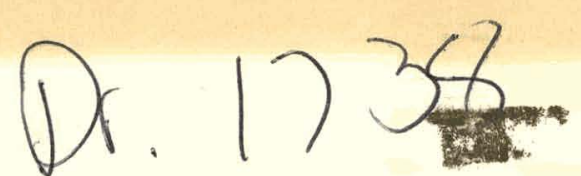

ORNL/TM-7407

\title{
On the Calculation of Similarity Solutions of Partial Differential Equations
}

L. Dresner

OPERATED BY

UNION CARBIDE CORPORATION FOR THE UNITED STATES DEPARTMENT OF ENERGY 


\section{DISCLAIMER}

This report was prepared as an account of work sponsored by an agency of the United States Government. Neither the United States Government nor any agency Thereof, nor any of their employees, makes any warranty, express or implied, or assumes any legal liability or responsibility for the accuracy, completeness, or usefulness of any information, apparatus, product, or process disclosed, or represents that its use would not infringe privately owned rights. Reference herein to any specific commercial product, process, or service by trade name, trademark, manufacturer, or otherwise does not necessarily constitute or imply its endorsement, recommendation, or favoring by the United States Government or any agency thereof. The views and opinions of authors expressed herein do not necessarily state or reflect those of the United States Government or any agency thereof. 


\section{DISCLAIMER}

Portions of this document may be illegible in electronic image products. Images are produced from the best available original document. 
Printed in the United States of America. Available from National Technical Information Service

U.S. Department of Commerce

5285 Port Royal Road, Springfield, Virginia 22161

NTIS price codes-Printed Copy: A03; Microfiche A01

This report was prepared as an account of work sponsored by an agency of the United States Government. Neither the United States Government nor any agency thereof, nor any of their employees, makes any warranty, express or implied, or assumes any legal liability or responsibility for the accuracy, completeness, or usefulness of any information, apparatus, product, or process disclosed, or represents that its use would not infringe privately owned rights. Reference herein to any specific commercial product, process, or service by trade name, trademark, manufacturer, or otherwise, does not necessarily constitute or imply its endorsement, recommendation, or favoring by the United States Government or any agency thereof. The views and opinions of authors expressed herein do not necessarily state or reflect those of the United States Government or any agency thereof. 
Contract No. W-7405-eng-26

FUSION ENERGY DIVISION

ON THE CALCULATION OF SIMILARITY SOLUTIONS

OF PARTIAL DIFFERENTIAL EQUATIONS

\section{I.. Dresner}

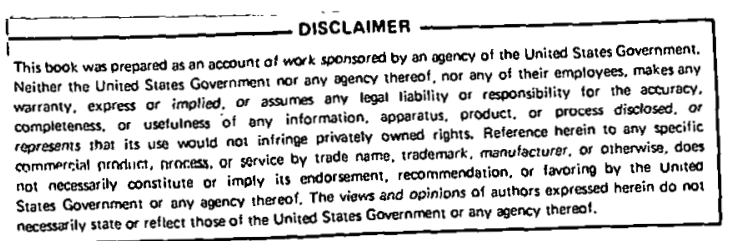

Date Published - August 1980

Prepared by the

OAK RIDGE NATIONAL LABORATORY

Oak Ridge, Tennessee 37830

operated by

UNION CARBIDE CORPORATION

for the

DEPARTMENT UF ENERGY 
THIS PAGE

\section{WAS INTENTIONALLY LEFT BLANK}




\section{CONTENTS}

ABSTRACT $\ldots \ldots \ldots \ldots \ldots \ldots \ldots \ldots \ldots \ldots \ldots \ldots \ldots \ldots \ldots \ldots \ldots \ldots \ldots \ldots \ldots \ldots$

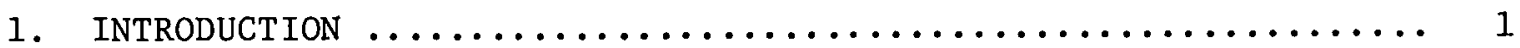

2. GROUP INVARIANCE OF THE ORDINARY DIFFERENTIAL

EQUATION $\ldots \ldots \ldots \ldots \ldots \ldots \ldots \ldots \ldots \ldots \ldots \ldots \ldots \ldots \ldots \ldots \ldots \ldots \ldots \ldots \ldots$

3. EXCEPTIONAL SOLUTIONS OF SECOND-ORDER EQUATIONS ............ 6

4. EXAMPLE: BLASIUS' ${ }^{\prime}$ EQUATION ........................

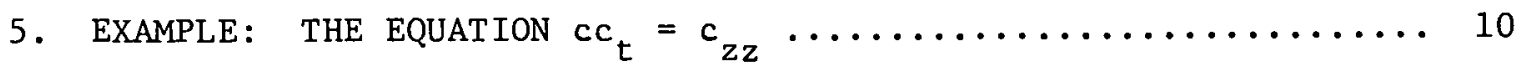

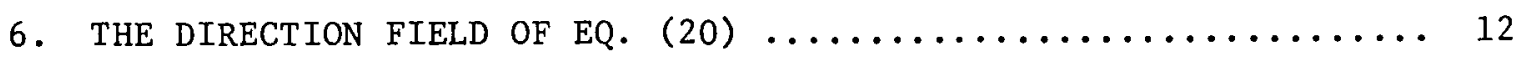

7. Calculation of THE SEPARATRIX ......................... 14

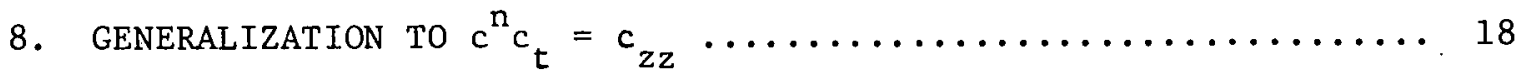

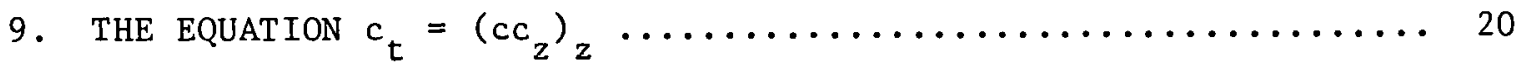

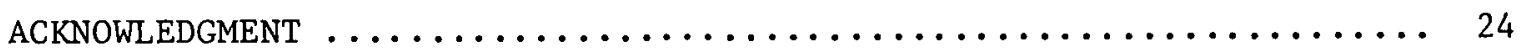

REFERENCES ...................................... 26 


\section{THIS PAGE}

\section{WAS INTENTIONALLY}

LEFT BLANK 


\section{ABSTRACT}

When a partial differential equation in two independent variables is invariant to a group $G$ of stretching transformations, it has similarity solutions that can be found by solving an ordinary differential equation. Under broad conditions, this ordinary differential equation is also invariant to another stretching group $G^{\prime}$, related to $G$. The invariance of the ordinary differential equation to $G^{\prime}$ can be used to simplify its solution, particularly if it is of second order. Then a method of Lie's can be used to reduce it to a first-order equation, the study of which is greatly facilitated by analysis of its direction field. The method developed here is applied to three examples: Blasius's equation for boundary layer flow over a flat plate and two nonlinear diffusion equations, $c c_{t}=c_{z z}$ and $c_{t}=\left(c_{z}\right)_{z}$. 


\section{INTRODUCTION}

Self-similar functions preserve their forms under certain scale transformations of their magnitudes and arguments. For example, the function $c=\exp \left(-r^{2} / 4 t\right) /(4 \pi t)^{3 / 2}$ preserves its form under the group of transformations $r^{\prime}=\lambda r, t^{\prime}=\lambda^{2} t, c^{\prime}=\lambda^{-3} c$, where $\lambda$ is any positive number. This function, as the reader may recognize, is the instantaneous point source solution of the spherically symmetrical diffusion equation $c_{t}=c_{r r}+2 c_{r} / r$. Its self-similarity arises from the invariance of the diffusion equation to the wider group of transformations $r^{\prime}=\lambda r, t^{\prime}=$ $\lambda^{2} t, c^{\prime}=\mu c$, where $\lambda$ and $\mu$ are posilive numbers independent of each other. Such self-similar solutions to partial differential equations are important because they are much easier to find than other solutions: when the partial differential equation has two independent variables, its self-similar solutions $c$ an be found by solving an ordinary differential equation.

According to Ames, 1 the study of self-similar solutions began in 1894 with Boltzman, ${ }^{2}$ who studied the diffusion equation $c_{t}=\left[D(c) c_{x}\right]_{x}$. As Ames also points out, Blasius's ${ }^{3} 1908$ solution of the boundary layer flow over a flat plate is self-similar. So are the 1941 solutions of Taylor ${ }^{4}$ and von Neumann ${ }^{5}$ for the pressure and flow fields created in air by a point explosion.

Birkhoff ${ }^{6}$ (1950) was the first explicitly to mention that invariance of a partial differential equation to a group of transformations (the word "group" now being used in its rigorous sense) could be used to find self-similar solutions. Since the time of Birkhoff's hint, self-similar solutions have been published to problems in diffusion, heat and mass transfer, hydrodynamics, shock propagation, solid mechanics, plasma physics, and applied superconductivity. ${ }^{7}$

Most applications involve one-parameter groups of stretching Linufurmations, i.e., transformations in which images are formed by multiplication by powers of the group parameter. Quite often, the ordinary differential equation to which the problem reduces is also invariant to a stretching group. This is no coincidence, and the conditions for it are explained below (Sect. 2). The group invariance 
of the ordinary differential equation can be exploited in two ways, the discussion of which forms the bulk of this paper. First, if the solution of the ordinary differential equation satisfies two-point boundary conditions, we can use the group invariance to find it without trial and error. This is illustrated below with Blasius's problem of boundary layer flow over a flat plate (Sect. 4). Second, if the ordinary differential equation is of second order, we can find, with the help of the group, new independent and dependent variables whose use reduces the equation to one of first order. ${ }^{8}$ This first-order equation can be analyzed by studying its direction field. $y$ The advantages of this reduction are illustrated below for the nonlinear diffusion equations $c c_{L}=c_{Z L}$ alld $c_{L}=\left(c c_{2}\right)_{L}$ (Bects. j-9). These examples make clear how important the group invariance of the ordinary differential equation is to the calculation of self-similar solutions. 


\section{GROUP INVARIANCE OF THE ORDINARY DIFFERENTIAL EQUATION}

Suppose we have a partial differential equation with one dependent variable $c$ and two independent variables $z$ and $t$. Suppose the partial differential equation is invariant to the family of one-parameter groups of transformations:

$$
\left.\begin{array}{l}
z^{\prime}=\lambda z \\
t^{\prime}=\lambda^{\beta} \\
c^{\prime}=\lambda^{\alpha} c
\end{array}\right\} \quad 0<\lambda<\infty .
$$

(Note that we lose no generality by taking the exponent of $\lambda$ in the first line equal to 1. )

If the partial differential equation is written in the primed variables and the substitution (1) made, it quite often happens that each term in the partial differential equation is multiplied by a power of $\lambda$. The exponents of $\lambda$ in these multipliers are linear combinations of $\alpha, \beta$, and 1 . Invariance means that the exponents of all terms are equal, and this equality leads to one or two independent linear equations in $\alpha$ and $\beta$. (There cannot be more than two independent linear equations because that would mean there is no solution for $\alpha$ and $\beta$ and contradict the assumed invariance of the partial differential equation.) If there are two linear relations, $\alpha$ and $\beta$ are uniquely determined, and the family of groups ( 1 ) reduces to a single group. If there is one linear relation, say,

$$
\mathrm{M} \alpha+\mathrm{N} \beta=\mathrm{L},
$$

then the partial differential equation is invariant to a one-parameter family of one-parameter groups. The groups of the family are labeled by $\alpha$ or $\beta$; the transformations of each group are labeled by $\lambda$. It is this second case that interests us.

To find self-similar solutions we look for solutions invariant to the group (1). The most general invariant relation connecting the 
variables $c, z$, and $t$ can be written: 10

$$
\frac{c}{t^{\alpha / \beta}}=y\left(\frac{z}{t^{1 / \beta}}\right)
$$

where $y$ is an arbitrary function. Substitution of (3) into the partial differential equation gives an ordinary differential equation that the function $y(x)$ must satisfy (here $x$ is an abbreviation for the argument $\left.z / t^{1 / \beta}\right)$. The values of $\alpha$ and $\beta$ are selected so that the boundary conditions can be satisfied. For example, if $c(0, t)=$ a prescribed constant, $\alpha=0$ and $\beta=\mathrm{L} / \mathrm{N}$. If $\int_{-\infty}^{+\infty} \mathrm{cd} z=$ a prescribed constant, $\alpha=-1$ and $\beta=(L+M) / N$. Denote by $\alpha_{0}$ and $\beta_{0}$ the values, satisfying (2), so selected. Then

$$
c=t^{\alpha_{0} / \beta_{0}} y\left(\frac{z}{1 / \beta_{0}}\right)
$$

is the most general relation connecting $c$, $a$, and $t$ that is invariant to group (1) of the family for which $\alpha=\alpha_{0}$ and $\beta=\beta_{0}$.

If we transform $c(z, t)$ given by (4) according to other groups of the family (1) for which $\alpha \neq \alpha_{0}$ and $\beta \neq \beta_{0}$, ite image $\varepsilon^{\prime}\left(z^{\prime}, t^{\prime}\right)$ must also satisfy the partial differential equation because the latter is invariant to all groups of the family ( 1$)$. We tind that

$$
c^{\prime}=\lambda^{\left(\alpha \beta_{0}-\alpha_{0} \beta\right) / \beta_{0}}\left(t^{\prime}\right)^{\alpha_{0} / \beta_{0}} y\left[\lambda^{\left(\beta \beta_{0}\right) / \beta_{0}} \frac{z^{\prime}}{\left(t^{\prime}\right)^{1 / \beta_{0}}}\right] \text {. }
$$

T.t. is easy to verify that

$$
\frac{\alpha \beta_{0}-\alpha_{0} \beta}{\beta_{0}-\beta}=\frac{L}{M}
$$


regardless of the values of $\alpha$ and $\beta$ as long as $\alpha$ and $\beta$ satisfy (2). Therefore, if we set $\lambda^{\left(\beta-\beta_{0}\right) / \beta_{0}}=\mu$ and drop the primes, (5) becomes

$$
c=t^{\alpha_{0} / \beta_{0}} \mu^{-L / M} y\left(\mu \frac{z}{1 / \beta_{0}}\right)=t^{\alpha_{0} / \beta_{0}} \mu^{-L / M} y(\mu x)
$$

The function $c(z, t)$ given by (7) is also a solution of the partial differential equation and is furthermore invariant to (1) when $\alpha=\alpha_{0}$ and $\beta=\beta_{0}$.

The one-parameter family of functions of $x, \mu^{-L / M} y(\mu x)$, appearing in (7), is the family of images of $y(x)$ under the one-parameter group

$$
\left.\begin{array}{l}
y^{\prime}=\mu^{L / M} y \\
x^{\prime}=\mu x
\end{array}\right\} 0<\mu<\infty
$$

[For $y^{\prime}\left(x^{\prime}\right) \equiv \mu^{L / M} y(x)=\mu^{L / M} y\left(x^{\prime} / \mu\right)$. If we replace $\mu$ by $1 / \mu$, this becomes $y^{\prime}\left(x^{\prime}\right)=\mu^{-L / M} y\left(\mu x^{\prime}\right)$.] A one-parameter family whose curves transform into each other under a group is said to be invariant to the group. Each function $y(x)$ satisfying (4) generates an invariant oneparameter family of functions $y(x)$ also satisfying (4), the invariance of the family being with respect to the group ( 8 ).

Suppose that the ordinary differential equation for $y(x)$ is of $n$th order. The solutions of such an equation form an $n$-parameter family of curves. From what we have just seen, this n-parameter family must decompose into an ( $n-1)$-parameter set of one-parameter families, each of which is invariant to (8). But then the entire n-parameter family is invariant to (8). This means that the differential equation for $y$ is also invariant to $(8)$, which is what we wanted to prove. 


\section{EXCEPTIONAL SOLUTIONS OF SECOND-ORDER EQUATIONS}

Lie $^{y}$ has given a prescription for finding the most general secondorder differential equation invariant to the group (8). If we introduce a group invariant $\mathrm{u}(\mathrm{x}, \mathrm{y})$ as a new independent variable and a firstdifferential invariant $v(x, y, \dot{y})$ as a new dependent variable, the most general second-order equation for $y$ in terms of $x$ has the form $d v / d u=$ $G(u, v)$, where $G(u, v)$ is some function of $u$ and $v$. An invariant and $a$ first-differential invariant of (8) are

$$
\left.\begin{array}{l}
u-\frac{y}{x^{a}} \\
v=\frac{\dot{y}}{x^{a-1}}
\end{array}\right\} a=\frac{L}{M} .
$$

Differentiation gives

$$
\begin{aligned}
d u & =(v-a u)\left(\frac{d x}{x}\right) \\
d v & =\left[\frac{\ddot{y}}{x^{a=2}}-(a-1) v\right]\left(\frac{d x}{x}\right) \\
& =|F(u, v)-(a-1) v|\left(\frac{d x}{x}\right)
\end{aligned}
$$

because Lie's theorem telìs us that $\ddot{y} / x^{a-2}$ must be a function $F(u, v)$ of $\mathrm{u}$ and $\mathrm{v}$. Thus

$$
\frac{d v}{d u}=\frac{\Gamma(11, v)-(a-1) v}{v-a u}
$$

Any integral curve $v(u)$ of (11) represents a first-order differential equation for $y$ in terms of $x$, i.e., a one-parameter family of integral curves $y(x)$ that transform into one another under the group (8). In addition to these one-parameter families, there are exceptional solutions 
arising from the singular points of (11). These exceptional solutions $y(x)$ correspond to constant values $u_{0}$ and $v_{o}$ of $u$ and $v$. Constant values of $u_{0}$ and $v_{0}$ mean that as $x$ and $y$ vary, $u$ and $v$ remain fixed at $u_{0}$ and $v_{0}$. Thus $d u=d v=0$, and we see then from (10) that $u_{0}$ and $v_{0}$ must satisfy the equations

$$
F\left(u_{0}, v_{0}\right)=(a-1) v_{0}
$$

and

$$
v_{0}=a u_{0}
$$

The only solutions of (12), of course, are the singular points of (11), assuming $F(u, v)$ is not itself singular. Thus the solution $y=u_{o} x^{a}$. corresponds to the singular point $\left(u_{0}, v_{0}\right)$. 
4. EXAMPLE: BLASIUS'S EQUATION

Blasius's equation for the stream function $c$ of the boundary layer developing along a flat plate can be written.

$$
c_{z} c_{z t}-c_{t} c_{z z}=c_{z z z}
$$

where $z$ measures distance transverse to the plate and $t$ measures distance along the plate. (Special units have been chosen in which the kinematic viscosity and the mean stream velocity are both equal to one.) Equation (13) is invariant to the group (1) if $\alpha-\beta=-1$, i.e., if $M=1, N=-1$, and $\mathrm{L}=-1$. The bundary cundicluns of Blaslus's problem are:

$$
\begin{aligned}
& c(0, t)=0, \\
& c_{z}(0, t)=0, \\
& c_{z}(\infty, t)=1, \\
& c_{t}(z, 0)=0 .
\end{aligned}
$$

Equatione (1/a) and (1/b) bccome $y(0)-0$ and $\dot{y}(0)-0$, rcopcctivcly: In order to satisfy (14c) we must have $\alpha=\alpha_{0}=1, \beta=\beta_{0}=2$. Then (14c) becomes $\dot{y}(\infty)=1$. Equation (14d) becomes $\lim _{x \rightarrow \infty}(y-x \dot{y})=0$ or what is the same thing, $\lim _{x \rightarrow \infty}(y / x)=A=$ a constant; this condition is the same as the condition $\dot{y}(\infty)=1$ if $A$ is chosen to be one. The differential equation for $y$ turns out to be

$$
2 \dddot{y}+y \ddot{y}=0 \text {, }
$$

and the boundary conditions again are

$$
y(0)=\dot{y}(0)=0
$$


and

$$
\dot{y}(\infty)=1
$$

The group (8) has the form

$$
\left.\begin{array}{l}
y^{\prime}=\frac{y}{\mu} \\
x^{\prime}=\mu x
\end{array}\right\}
$$

in this example. It is easy to verify that (15a) is invariant to (16). The boundary conditions (15b) and (15c) refer respectively to $x=0$ and $x=\infty$. Neither (15b) nor (15c) alone is sufficient to allow numerical solution of (15a). Ordinarily, we would assume a value of $\ddot{y}(0)$, integrate $(15 a)$ to large $x$, find $\dot{y}(\infty)$, correct $\ddot{y}(0)$, and repeat. We can avoid repetition by using the relation $\dot{y}^{\prime}=\dot{y} / \mu^{2}$ to find the value of $\mu$ that will make $\dot{y}^{\prime}(\infty)=1$. Then using (12) we can find $y^{\prime}\left(x^{\prime}\right)$ by scaling the function $y(x)$ calculated in the first numerical integration. If high accuracy is sought, much repetition can be avolded in this way. 
5. RXAMPIF: THF EQUATION $\mathrm{cc}_{t}=\mathrm{c}_{z z}$

The equation $c c_{t}=c_{z z}$ arises in the problem of transient heat transfer from a heated surface to a single-phase, near-critical fluid and in the problem of the expulsion of fluid from a long, slender, heated tube. In both of these problems the boundary and initial conditions are $c(z, 0)=0, c(\infty, t)=0$, and $c_{z}(0, t)=-b$, a prescribed constant. These are the boundary conditions we shall consider here.

The partial differential equation is invariant to the group (1) if $\alpha-\beta=-2$, i.e., if $M=1, N=-1$, and $L=-2$. In order to satisfy the boundary condition that $c(0, t)$ is constant, $\alpha$ must be chosen equal to $\alpha_{0}=1$; then $\beta=\beta_{0}=3$. For convenience, we introduce a factor of $\sqrt{3}$ into the definition of $x$, i.e., take $c=t^{1 / 3} y\left(z / \sqrt{3} t^{1 / 3}\right)=t^{1 / 3} y(x)$. Then

$$
\ddot{y}=y(y-x \dot{y})
$$

and

$$
y(m)=0, \quad \dot{y}(0)=-b \sqrt{3} .
$$

The group (8) now takes the form

$$
\left.\begin{array}{l}
y^{\prime}=\mu^{-2} y \\
x^{\prime}=u x
\end{array}\right\},
$$

and, as expected, the ordinary differential equation (17a) is invariant to it. According to the theorem of Lie, ${ }^{8}$ if we introduce as new independent and dependent variables a group invariant $u(x, y)$ and a firstdifferential group invariant $v(x, y, \dot{y})$, Eq. (17a) will become a firstorder differential equation for $v$ in terms of $u$. Analysis of the direction field of this first-order equation can tell us much about the solutions of (18a); in this problem it will give us the value of $y(0)$ at the cost of a single integration. 
The choice of $u$ and $v$ is not unique; a convenient choice is

$$
u=x^{2} y
$$

and

$$
v=x^{2}(y-i x \dot{y})
$$

Using (17) we find that

$$
\frac{d v}{d u}=\frac{v(2-u)}{3 u-v}
$$


6. THE DIRECTION FIELD OF EQ. (20)

Shown in Fig. 1 is the direction field of Eq. (20). The slope $\mathrm{dv} / \mathrm{du}$ vanishes on the lines $\mathrm{L}_{1}: \mathrm{v}=0$ and $\mathrm{L}_{2}: \quad \mathrm{u}=2$ and is infinite on the line $\mathrm{L}_{3}: \mathrm{v}=3 \mathrm{u}$. There are two singular points, $0:(0,0)$ and $P$ : $(2,6)$. The singular point $P$ is a saddle point; the origin 0 is a node. Traversing $P$ are two separatrices $S_{1}$ and $S_{2}$. The separatrix $S_{1}$ also traverses the singular point 0 . Some typical integral curves are also shown in Fig. 1.

The family of integral curves of Eq. (17) we are seeking transforms into itself under the transformations of the group (18). In other words, the image of each curve of the family is another curve of the family. The family thus corresponds to a single integral curve in the $(u, v)$ plane. Furthermore, this single curve must pass through the origin 0 because $u$ and $v$ both approach zero as $x$ approaches zero with $y$ and $\dot{y}$ remaining finite. Of the integral curves passing through the origin, the separatrix $S_{1}$ is the one we want. For in the neighborhood of the singular point $\mathrm{P}, \mathrm{y} \cong 2 / \mathrm{x}^{2}$, and this is a satisfactory asymptotic behavior for the integral curve we are seeking. 
ORNL-DWG $80-2740$ FED

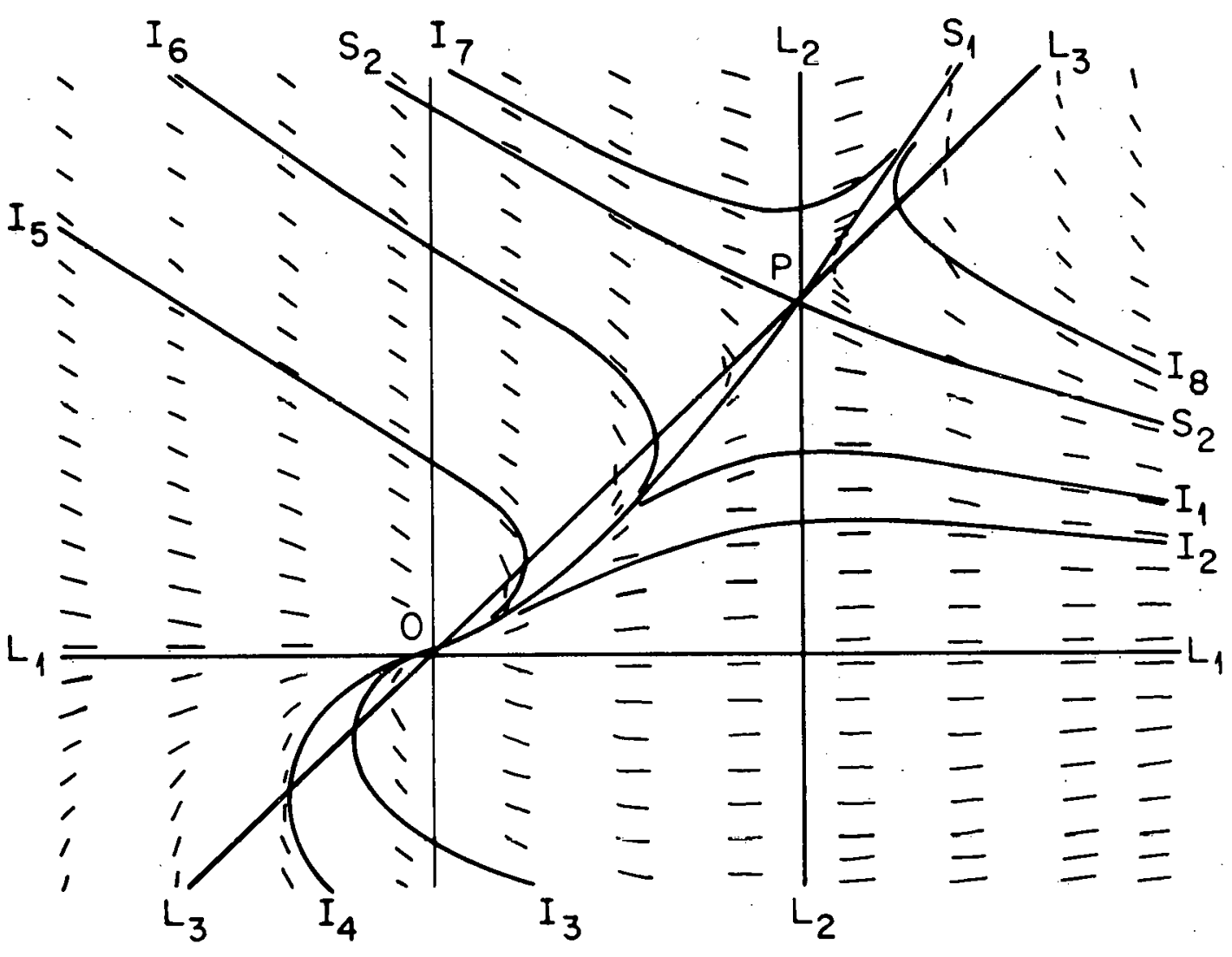

Fig, 1. The direction field of Eq. (20), 0 and $P$ are the singular points, $S_{1}$ and $S_{2}$ are the separatrices, and the curves marked with. I are integral curves. 


\section{CALCULATION OF THE SEPARATRIX}

Near the origin where $u \ll 2$, Eq. (20) can be approximated by the homogeneous equation $\mathrm{dv} / \mathrm{du}=2 \mathrm{v} /(3 \mathrm{u}-\mathrm{v})$. By "homogeneous" we mean that the differential equation is invariant to the group $v^{\prime}=\lambda v, u^{\prime}=\lambda u$. The separatrix $S_{1}$, being an invariant curve of the group, must have the form $v=m u$. Substitution into the homogeneous differential equation shows that $\mathrm{m}=1$.

If we substitute $v=u+w$ into $(20)$, then to lowest order $\mathrm{dw} / \mathrm{du}=$ $3 w / 2 u$, so that $w=\mathrm{Lu}^{3 / 2}$. l'his suggestes that $v$ may be expanded $1 \mathrm{n}$ powers of $\mathrm{u}^{1 / 2}$ near the origin. If we set

$\mathrm{v}=\mathrm{u}+\mathrm{Cu}^{3 / 2}+\mathrm{Du}^{2}+\mathrm{Eu}^{5 / 2}+\mathrm{Fu}^{3}+\mathrm{Gu}^{7 / 2}+\mathrm{Hu}^{4}+\mathrm{Ju}^{9 / 2}+\mathrm{Ku}^{5}+\ldots$

substitute into (20), clear fractions, and equate coefficients of equal powers of $u$, we get

$$
\begin{aligned}
& D=\frac{3}{2} C^{2}-1, E-\frac{1}{2}\left(\frac{7}{2} C D-C\right), \quad F-\frac{1}{3}\left(4 C E+2 D^{2}-D\right), \\
& G=\frac{1}{4}\left[\frac{9}{2}(D E+F C)-E\right], \quad H=\frac{1}{5}\left[\frac{5}{2} E^{2}+5(F D+G C)-F\right], \\
& J=\frac{1}{6}\left[\frac{11}{2}(F E+G D+C H)-G\right], \\
& K=\frac{1}{7}\left[3 F^{2}+6(G E+H D+C J)-H\right] .
\end{aligned}
$$

From Eq. (22) it is clear that once we fix C, all the higher coefficients in (21) are determined. Since all the integral curves passing through the origin are tangent to one another (and to the line $v=u$ ), it is clear that they are distinguished from one another by the value of $\mathrm{C}$. Finally, 


$$
c=\lim _{u \rightarrow 0}\left(\frac{v-u}{u^{3 / 2}}\right)=-\frac{\dot{y}(0)}{[y(0)]^{3 / 2}}
$$

A similar procedure near the singular point P: $(2,6)$ gives for the separatrix $S_{1}$

$$
\begin{aligned}
& t=A s+B s^{2}+R s^{3}+S s^{4}+\ldots, \\
& t=v-6, \quad s=u-2, \\
& A=\frac{1}{2}(3+\sqrt{33}), \quad B=\frac{A}{3 A-6}, \\
& R=\frac{B(1-2 B)}{4 A-9}, \quad s=\frac{L(1-5 B)}{5 A-12} .
\end{aligned}
$$

We find the value of $C$ on $S_{1}$ by using (24) to advance a short distance along $S_{1}$ away from $P$. Then we integrate (20) numerically, advancing along $S_{1}$ towards 0 . When we get close to 0 , we match the numerical solution to the series (21) by choosing $\dot{C}$ correctly. In this way, with a single numerical integration, we find

$$
C=0.932 \text {. }
$$

This numerical integration, as well as all others mentioned later, was performed by the fourth-order Runge-Kutta method on a programmable desk calculator (Hewlett-Packard 97). Once the value of $C$ is in hand, calculation of $\mathrm{y}$ is an easy matter because consistent initial values can be obtained from (23). Figure 2 shows the curve of $y$ versus $x$ for which $y(0)=1$. As expected, $y \sim 2 / x^{2}$ for large $x$. Also shown is the following simple analytic approximation $y$ :

$$
y \cong\left(1+c x+\frac{x^{2}}{2}\right)^{-1}
$$




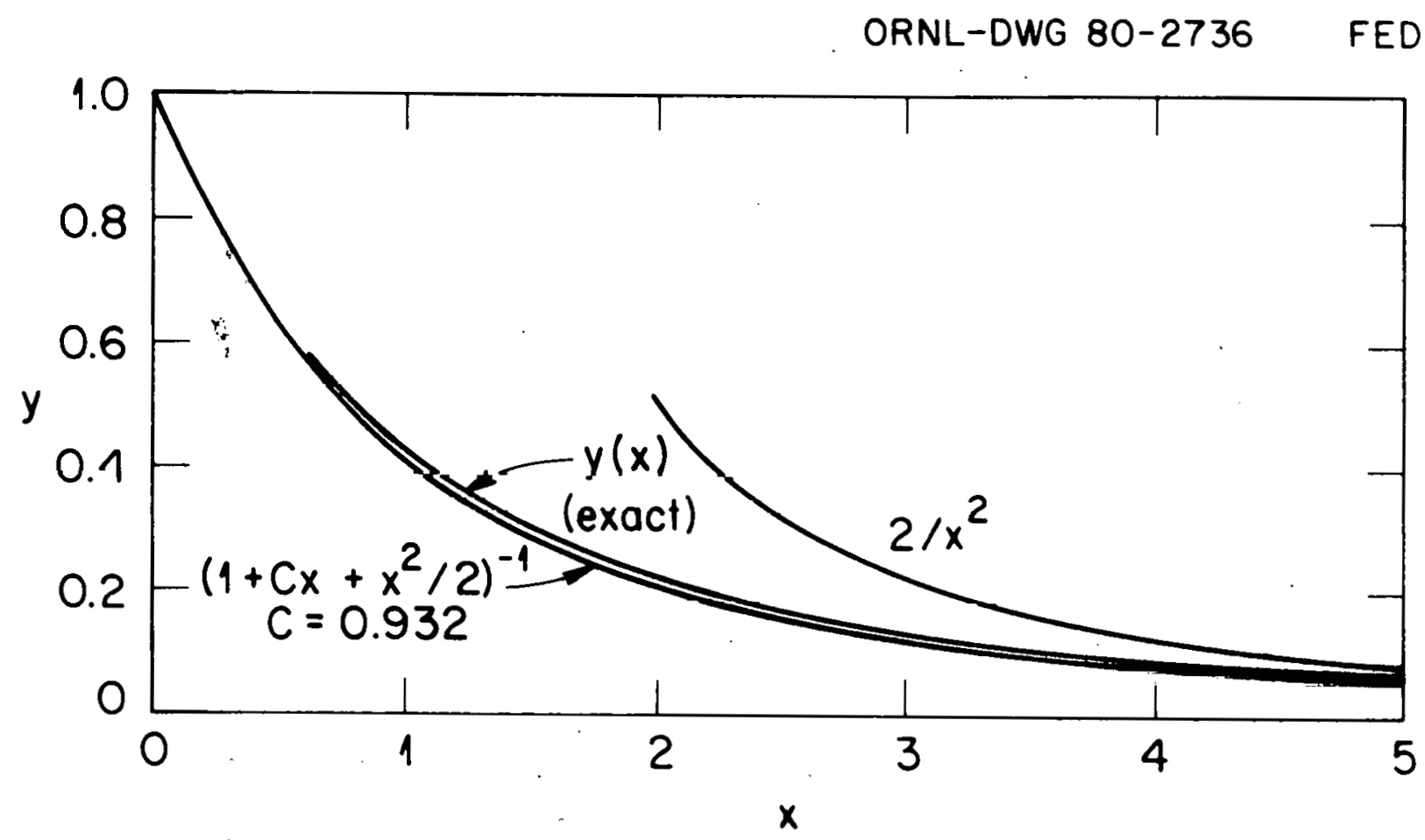

Fig. 2. The function $y(x)$ in Sect. 7 for the case $y(0)=1$. 
for which $y(0)=1$ and $\dot{y}(0)=-C$. It follows from (18) that in general

$$
y \cong \lambda^{2}\left(1+\lambda C x+\frac{\lambda^{2} x^{2}}{2}\right)^{-1}
$$

Finally,

$$
\frac{c(0, t)}{t^{1 / 3}}=1.51 \mathrm{~b}^{2 / 3}
$$


8. GENERALIZATION TO $c^{n} c_{t}=c_{z z}$

The partial differential equation $c^{n} c_{t}=c_{z z}$ can be treated in the same way when $n \neq 1$ as when $n=1$. In general, $n \alpha-\beta=-2$ so that $M=n, N=-1$, and $L=-2$. To satisfy the boundary condition $c_{z}(0, t)=-b$, a prescribed constant, we must take $\alpha=\alpha_{0}=1$ and $\beta=\beta_{0}=n+2$. If we set $c=t^{1 / \beta_{0}} y\left(z / \sqrt{\beta_{0}} t^{1 / \beta_{0}}\right)$, we find $\ddot{y}=y^{n}(y-x \dot{y}), \dot{y}(0)=-b \sqrt{\beta_{0}}$, and $y(\infty)=0$ as before. As expected, the differential equation for $y$ is invariant to the group $y^{\prime}=\mu^{-2 / n} y, x^{\prime}=\mu x$. If we choose $u=x^{2 / n} y$ and $v=x^{2 / n}(y-x \dot{y})$ we find

$$
\frac{d v}{d u}=\frac{v\left(2-n u^{n}\right)}{(n+2) u-n v}
$$

The direction field of (26) is similar to that of Fig. 1. There is a right triangle formed by lines $\mathrm{L}_{1}, \mathrm{~L}_{2}$, and $\mathrm{L}_{3}$ in the $(\mathrm{u}, \mathrm{v})$ plane like the one formed by $L_{1}, L_{2}$, and $L_{3}$ in Fig. 1. On $L_{3}:(n+2) u=n v$ the slope is infinite; on $\mathrm{L}_{2}: \mathrm{u}=(2 / \mathrm{n})^{1 / \mathrm{n}}$ and $\mathrm{L}_{1}: \mathrm{v}=0$ the slope is zero. The two vertices on the line $\mathrm{L}_{2}$ are singular points. These pointes are $0:(0,0)$ and $P:\left[(2 / 1)^{1 / n},(1 / 2 / n)(2 / n)^{1 / n}\right]$. They are joined by a separatrix that near the origin has the form

$$
v=u+c_{n} u^{(n+2) / 2}+\ldots
$$

sũ that

$$
C_{n}=\lim _{u \rightarrow 0}\left[\frac{v-u}{u(n+2) / 2}\right]=\frac{-\dot{y}(0)}{[y(0)]^{(n+2) / 2}}
$$

Finally, the separatrix near $P$ corresponds to asymptotic behavior of $y(x)$ of the form 


$$
\begin{aligned}
& y \sim\left(\frac{2}{n}\right)^{1 / n} x^{-2 / n}, \\
& \dot{y} \sim-\left(\frac{2}{n}\right)^{1+1 / n} x^{-1-2 / n} .
\end{aligned}
$$

When $n=2$, I used the power series method to calculate $C_{2}$ and found $\mathrm{C}_{2}=0.777$. When $\mathrm{n}=0$, the ordinary differential equation $\ddot{y}=y-x \dot{y}$ is solved by

$$
y=e^{-x^{2} / 2}-x \int_{x}^{\infty} e^{-x^{2} / 2} d x
$$

It follows from (33) that $y(0)=1$ and $\dot{y}(0)=-\sqrt{\pi / 2}$. Thus $c_{0}=\sqrt{\pi / 2}=$ 1.253: Fina11y,

$$
\frac{c(0, t)}{t^{1 /(n+2)}}=\left(\frac{\sqrt{n+2}}{c_{n}}\right)^{2 /(n+2)} b^{2 /(n+2)}
$$

Knowing $C_{0}, C_{1}$, and $C_{2}$, we can interpolate to find $C_{n}$ for intermediate values. It is worth remarking that when $n \geqslant 2, \int_{0}^{\infty} y d x$ diverges, a fact which may be of importance in applications. 
9. THE EQUATION $\check{C}_{t}=\left(\mathrm{CC}_{z}\right)_{z}$

This partial differential equation $c_{t}=\left(c c_{z}\right)_{2}$ is invariant to the group (1) if $\alpha+\beta=2$, i.e., if $M=1, N=1$, and $L=2$. If we set

$$
c=t^{\alpha / \beta} y\left(\frac{z}{t^{1 / \beta}}\right)
$$

we find the following ordinary differential equation for $y$ :

$$
\beta(\underline{y y})^{\bullet}=\alpha y-x \dot{y} \cdot
$$

Equation (36) is invariant to the group $y^{\prime}=\mu^{2} y, x^{\prime}=\mu x$ as expected. If we set $u=y / x^{2}$ and $v=\dot{y} / x$ we obtain the first-order equation:

$$
\frac{d v}{d u}=\frac{\alpha u-v-\beta v^{2}-\beta v u}{\beta u(v-2 u)}
$$

The choice of $\alpha$ and $\beta$ depends on the boundary conditions. Some typical boundary conditions together with the corresponding values of $\alpha$ and $\beta$ are as follows:

1. Clamped temperature: $c(0, t)$ constant

$$
\begin{array}{ll}
\alpha=0, & \beta=2 \\
\alpha=\frac{1}{2}, & \quad \beta=\frac{3}{2} \\
\alpha=1, & \beta=1 \\
\alpha=-1, & \beta=3
\end{array}
$$

2. Clamped flux: $\left(c c_{z}\right)_{z=0}$ constant

3. Ramped temperature: $c(0, t) \sim t$

4. Point source: $\int_{-\infty}^{+\infty} \operatorname{cdz}$ constant

Let uo firct consider case 4, for wh1ch (37) becomes

$$
\frac{d v}{d u}=-\frac{(u+v)(1+3 v)}{3 u(v-2 u)}
$$


The direction field of (38) is shown in Fig. 3. The slope $\mathrm{dv} / \mathrm{du}$ vanishes on the lines $L_{l}: u+v=0$ and $L_{2}: v=-1 / 3$ and is infinite on the lines $\mathrm{L}_{3}: \mathrm{u}=0$ and $\mathrm{L}_{4}: \mathrm{v}=2 \mathrm{u}$. There are three singular points, 0 : $(0,0), P:(0,-1 / 3)$, and $Q:(-1 / 6,-1 / 3)$. It is immediately evident from the figure that $v=-1 / 3$ solves (38). Thus

$$
\dot{y}=-\frac{x}{3}
$$

and

$$
y=\frac{\left(x_{0}^{2}-x^{2}\right)}{6},
$$

a solution found earlier by Pattle.11 Solution (39) enables us to satisfy the requirement that $y$ be zero at infinity by making y vanish for $x \geqslant x_{0}$. For $x<x_{0}$, we use (39b). Interestingly, there is no solution $y(x)$ for which $y$ and $\dot{y}$ approach zero continuously as $x$ approaches infinity. Such a solution would correspond to an integral curve in the $(u, v)$ plane passing through the origin. However, none of the integral curves that do so ever attains the limit $u \rightarrow \infty$.

Let us now turn to case 2 . Then (37) becomes

$$
\frac{d v}{d u}=\frac{u-2 v-3 v^{2}-3 u v}{3 u(v-2 u)}
$$

Figure 4 shows the direction field of (40). The slope $\mathrm{dv} / \mathrm{du}=0$ on the curve $\mathrm{C}: \mathrm{u}=\mathrm{v}(2+3 \mathrm{v}) /(1-3 \mathrm{v})$. (The curve $\mathrm{C}$ has two branches, one of which is shown in Fig. 4. The other branch is in the second quadrant and is of no concern to us here,) The slope $\mathrm{dv} / \mathrm{du}=\infty$ on the lines $\mathrm{L}_{1}$ : $\mathrm{u}=0$ and $\mathrm{L}_{2}: \mathrm{v}=2 \mathrm{u}$. There are three singular points, $0:(0,0), \mathrm{P}:$ $(0,-2 / 3)$, and $Q:(-1 / 6,-1 / 3)$, and two separatrices, $S_{1}$ and $S_{2}: v=u / 2$. The singular point $Q$ leads to the exceptional solution $y=-x^{2} / 6$ and the separatrix $S_{2}$ leads to the family of solutions $y=$ constant $\sqrt{x}$. Neither of the solutions is of any use to us here since neither is ever zero for any $x>0$. 


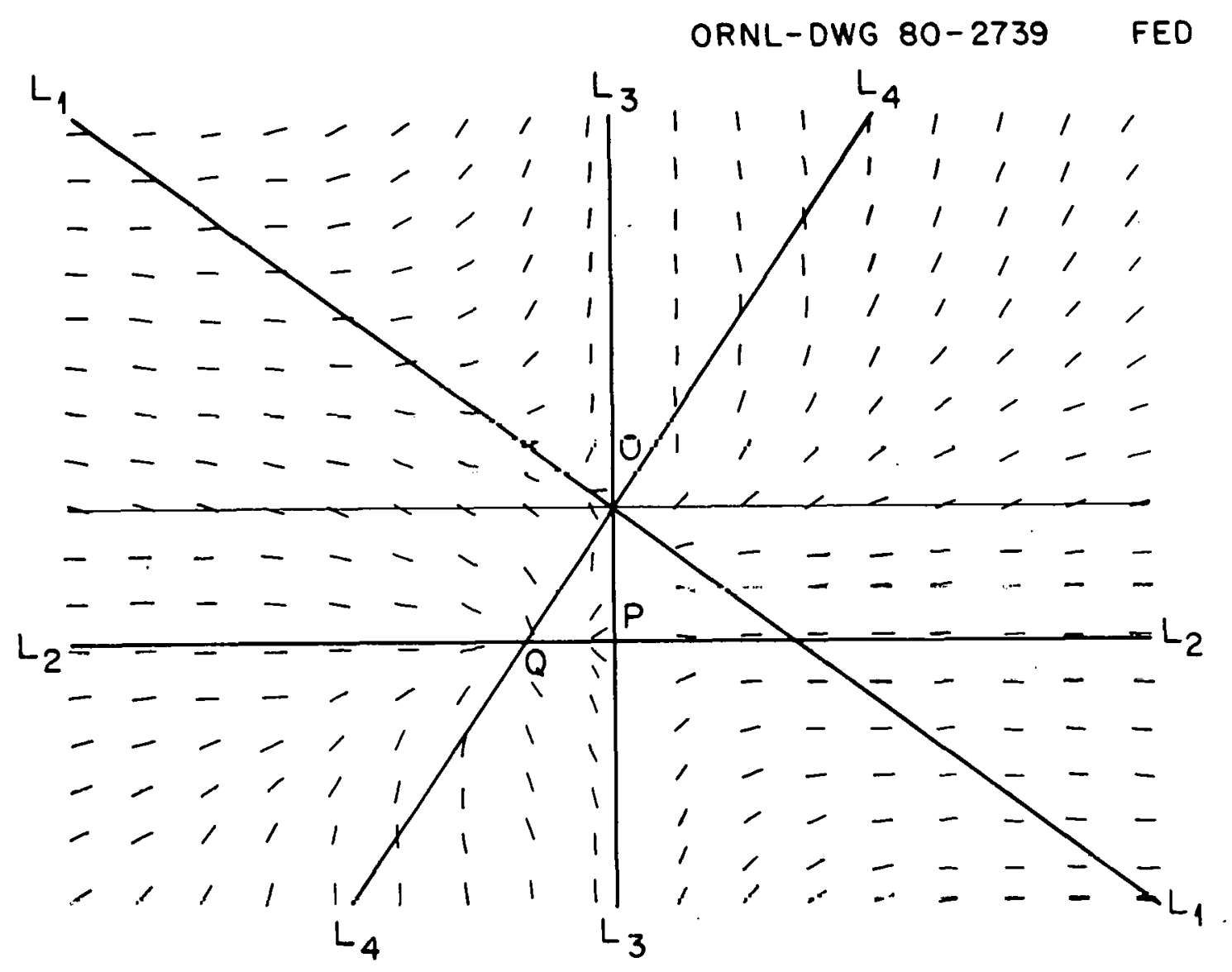

F1g. 3. The direction fleld of Eq. (38). 


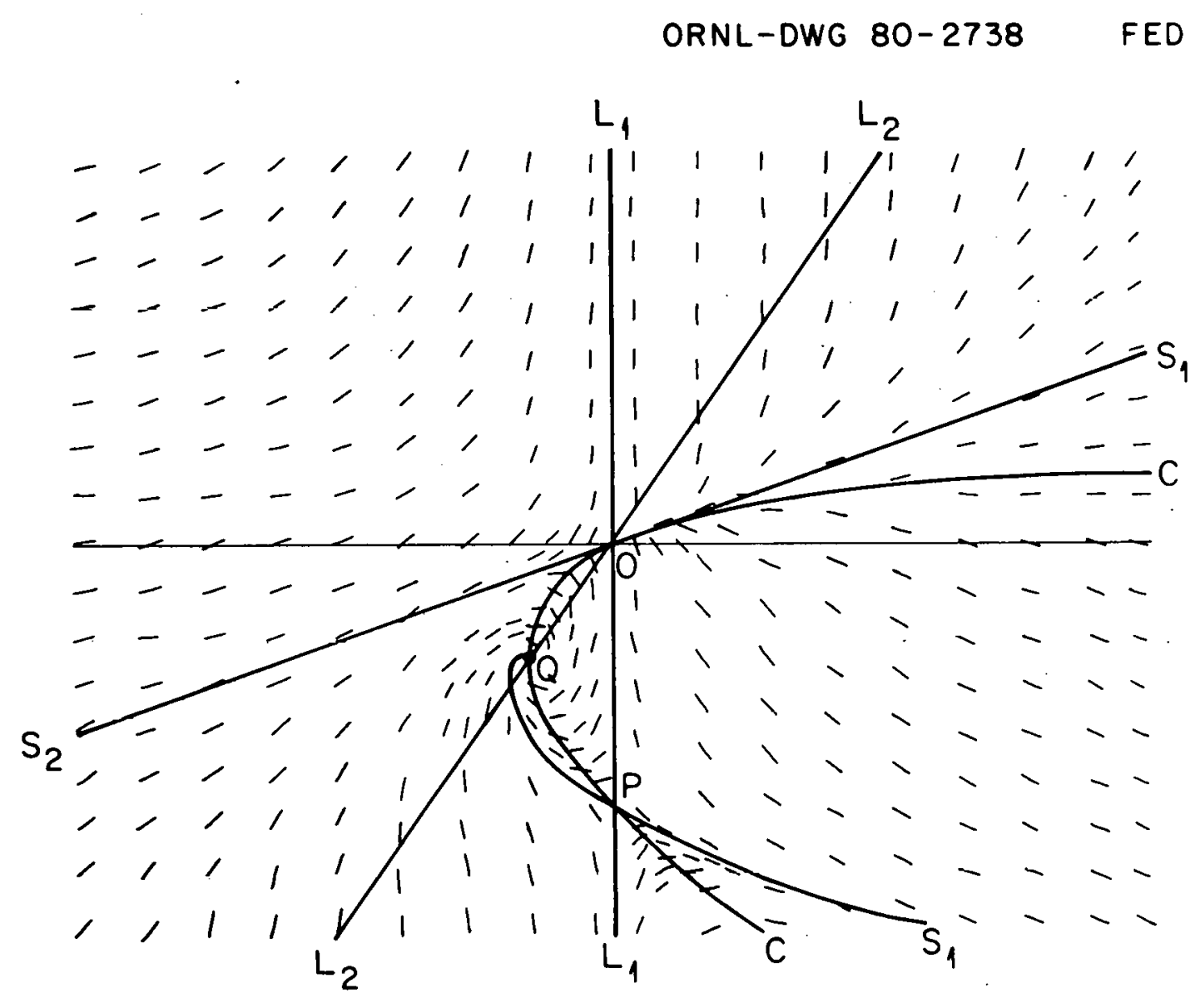

Fig. 4. The direction field of Eq. (40). 
There is no solution for which $y$ and $\dot{y} \rightarrow 0$ as $x \rightarrow \infty$. Such a solution must correspond to an integral curve in the $(u, v)$ plane passing through the origin. All such curves are tangent to $S_{2}$ near the origin. and thus behave asymptotically like $\sqrt{x}$ for large $x$. If we look for solutions $y(x)$ that have a root at some $x=x_{0}$, then at $x_{0}, y=0$ and $\dot{y}<0$ so that $u=0, v<0$. The only point that fills the bill is the singular point $P$, and the family of curves $y(x)$ we are seeking corresponds to the separatrix $S_{1}$. The value of $v$ at the point $P$ gives the slope $\dot{y}\left(x_{0}\right)=-2 x_{n} / 3$, and knowing this slope, we can undertake a numerical integration to find $y(x)$. The results of such an integration for the case $x_{0}=1$ are shown in Fig. 5. Other cases can be obtained by transformation with the group $y^{\prime}=\mu^{2} y, x^{\prime}=\mu x$.

When $u$ is large and positive, the separatrix $S_{1}$ has the asymptotic form $v=-C \sqrt{u}$, where $C$ is a constant. If we substitute the definitions of $u$ and $v$ in this equation, we find $\dot{y}=-\sqrt{y}$. Since $u \rightarrow \infty$ as $x \rightarrow 0$, this means

$$
\frac{-\dot{y}(0)}{\sqrt{y(0)}}=c \text {. }
$$

The numerical integration of (36) used to draw Fig. 5 gave $C=0.679$; $a$ numerical integration of (40) starting on $S_{1}$ near $P$ gave the same result. At this point we break off further discussion of examples.

\section{ACKNOWLEDGMENT}

I wish to express my gratitude to J. K. Ballou for writing the computer graphics program with which Figs. 1, 3, and 4 were produced. 


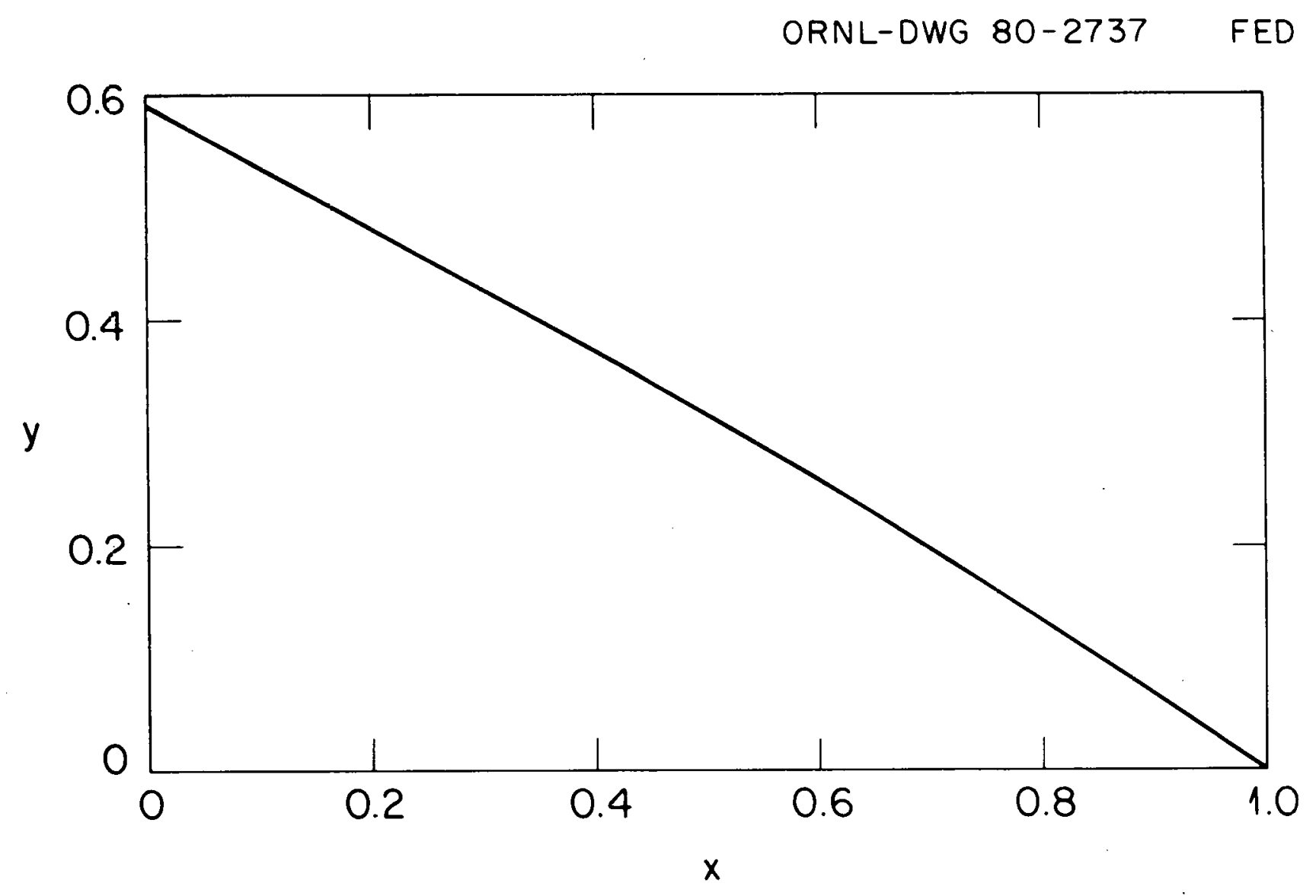

Fig. 5. The solution $y(x)$ of Eq. (36) for case 2: $\alpha=1 / 2, \beta=3 / 2$ when $x_{0}=1$ 


\section{REFERENCES}

1. W. F. Ames, "Some Ad-hoc Techniques for Nonlinear Partial Differential Equations," in Mathematical Physics and Physical Mathematics, Vol. 2, ed. by K. Maurin and R. Raczka, D. Reidel Publishing Co., Boston, 1974 .

2. L. Boltzmann, Ann. Physik (N.F.) 53, 959 (1894).

3. H. Blasius, Z. Angew. Math. Phys. 56, 1 (1908).

4. G. Taylor, Ministry of Home Security, R.C. 210 (1941); published later in Proc. R. Soc. London A201, 159 (1950).

J. J. voon licumann, "ghuule Wibul 3 Lablud by au Infiniteaimally fihnrt Detonation of Given Energy," National Defense Research Committee, Division B, June 30, 1941.

6. G. Birkhoff, Hydrodynamics, Princeton Univ. Press, Princetón, 1950.

7. For a short bibliography of this literature, see Sect. 8 of Ref. 1.

8. A. Cohen, An Introduction to the Lie Theory of One-Parcometer Groups, Sect. 27, G. E. Stechert and Co., New York, 1931.

9. L. Dresner, J. Math. Phys. 12(7), 1339 (1971).

10. See Sect. 11 of Ref. 8 .

11. R. E. Pattle, Q. J. Mech. Appl. Math. XII (4), 407 (1959). 
ORNL/TM-7407

Dist. Category UC-20 b

\section{INTERNAL DISTRIBUTION}

$\begin{aligned} & \text { 1. } \text { J. K. Ballou } \\ & \text { 2. } \text { D. B. Batchelor } \\ & \text { 3. } \text { R. A. Dory } \\ & \text { 4-8. } \text { L. Dresner } \\ & 9 . \text { C. W. Kee } \\ & \text { 10. } \text { J. Sheffield } \\ & \text { 11. A. Solomon } \\ & \text { 12. } \text { D. A. Spong } \\ & \text { 13. } \text { W. C. T. Stoddart } \\ & 14 . \text { R. E. Textor }\end{aligned}$

15-16. Central Research Library

17. Document Reference Section

18-19. Laboratory Records Department

20. Laboratory Records, ORNL-RC

21. Fusion Energy Division Reports Office

22-23. Fusion Energy Division Library

24. ORNL Patent Office

\section{EXTERNAL DISTRIBUTION}

25. E. Adam, Airco, Inc., 100 Mountain Avenue, Murray Hil1, NJ

26. Y. Aiyama, Electrotechnical Laboratory, 5-4-1 Mukodai-cho, Tanashi-City, Tokyo, Japan

27. D. J. Anthony, General Electric Company, Building 23, Room 290, 1 River Road, Schenectady, NY 12345

28. V. D. Arp, National Bureau of Standards, Boulder, CO 80302

29. M. P. G. Avanzini, Nucleare Italiana Reattori Azanzati, NIRA S.P.A., Piazza Carignano, 2-16128 Genova, Casella Postale N. 1166,16100 Genova, Italy

30. G. Bogner, Forschungslaboratorien, Siemens Aktienge Sellshaft, Postfach 325, D8520 Erlanger 2, Federal Republic of Germany

31. A. Clark, National Bureau of Standards, Boulder, CO 80302

32. D. L. Coffey, American Magnetics, Inc., P.O. Box R, Oak Ridge, TN 37830

33. E. W. Collings, Battelle Memorial Institute, 505 King Avenue, Columbus, $\mathrm{OH} 43201$

34. R. W. Conn, Department of Chemical, Nuclear, and Thermal Engineering, University of California at Los Angeles, Los Angeles, CA 90024

35. H. Desportes, STIPE, CEN/Saclay, F91190, Gif-sur-Yvette, France

36. G. W. Donaldson, School of Electrical Engineering, University of New South Wales, P.0. Box 1, Kensington, New South Wales 2033, Australia

37. R. W. Fast, Manager, Experimental Facilities, Fermi National Accelerator Laboratory, P.0. Box 500, Batavia, IL 60510

38. F. Fickett, National Bureau of Standards, Boulder, CO 80302

39. J. File, Princeton University, Plasma Physics Laboratory, Princeton, NJ 08540

40. H. K. Forsen, Laser Enrichment Department, Exxon Nuclear Company, Inc., 777 106th Avenue, NE, C-00777, Bellevue, WA 98009 
41. H. P. Furth, Princeton University, Plasma Physics Laboratory, Princeton, NJ 08540

42. Y. Furuto, Chief of Superconducting Group, Central Research Laboratory, Furukawa Electric Company, Ltd., 9-15, 2-Chome, Futuba, Shinagawa-ku, Tokyo 141, Japan

43. W. F. Gauster, c/o H. Kirchmayr, Director, Institut für Experimentalphysik, der Technischen Hochschule in Wien, A-1040 Vienna, Karlsplatz 13, Austria

44. E. Gregory, Airco, Inc., 100 Mountain Avenue, Murray Hi11, NJ 07974

45. D. S. Hackley, General Dynamics, Convair Division, P.0. Box 80847 , San Diego, CA 92138

46. R. Hancox, UKAEA, Culham Laboratory, Abingdon, Oxon, OX14 3DB, England

47. W. Heinz, Gesellschaft für Kernforschung, Institut für Experimental Kernphysik, 75 Karlsruhe, Postfach 3640, Federal kejublic of termany

48. C. D. Henning, Lawrence Livermore Laboratory, Y.U. Bóx 8ü8, L-533, Livermore, CA 94550

49. T. Hiraoka, Japan Atomic Energy Research Institute, Tokai Research Establishment, Tokai-Mura, Naka-Gun, Ibaraki-Ken, Japan

50. R. L. Hirsch, Deputy Manager, Science and Technology Division, Exxon Corporation, 1251 Avenue of the Americas, New York, NY 10020

51. M. O. Hoenig, Massachusetts Institute of Technology, National Magnet Laboratory, 170 Albany Street, Cambridge, MA 02139

52. M. Iwamoto, Central Research Laboratory, Mitsubishi Electric Corporation, 80 Nakano, Minamishimizu, Anagasaki, Hyogo Prefecture, Japan 660

53. C. K. Jones, Manager, Cryogenic Research Laboratory, Westinghouse Electric Corporation, Reesarch and Development Senter, Pittshurgh, FA 1.5235

54. A. Knobloch, Max-Planck-Institut für Plasmaphysik, Abteilung Technik, 8046 Garching bei München, Federal Republic of Germany

55. P. Komarek, Institut für Experimental Kernphysik, 75 Karlsruhe, Postfach 3640 , Federal Republic of Germany

56. K. Koyama, Electrotechnical Laboratory, 5-4-1 Mukodai-cho, TanashiCity, Tokyo, Japan

57. K. Kuroda, Hitachi, Ltd., Central Research Laboratory, 1-280, Higa Shiko Igakubo, Kokubumji, Tokyo 185, Japan

58. J. C. Lottin, CEN/Saclay, Dep. du Synchrotron Saturne, B.'. $.2,91$ Gif-sur-Yvette, 91190, France

59. Max-Planck-Institut für Plasmaphysik, Hauptbibliothek, 8046 Garching bei München, Federal Republic of Germany

60. F. Moon, Department of Theoretical and Applied Mechanics, Cornell University, Ithaca, NY 14850

61. T. Ogasawara, Department of Physics, College of Science and Engineering, Nihon University, Kanada-Surugadai, Chiyoda-ku, Tokyo, Japan

62. H. Ogiwara, Toshiba Research and Development Center, 1 Komukai Tsohibacho, Saiwai-ku, Kawasaki-City, Kanagawa 210, Japan

63. J. Parain, CEN/Saclay, B.P. 2, F91190, Gif-sur-Yvette, France

64. J. R. Powell, Brookhaven National Laboratory, Upton, NY 11973 
65. P. Reardon, Princeton University, Plasma Physics Laboratory, P.0. Box 451, Princeton, NJ 08540

66. R. P. Reed, National Bureau of Standards, Boulder, CO 80302

67. D. J. Rose, Department of Nuclear Engineering, Massachusetts Institute of Technology, Cambridge, MA 02139

68. G. Sacerdoti, Laboratorio Nazionali, Cas. Postale 70, 00044 Frascati, Italy

69. W. B. Sampson, Brookhaven National Laboratory, Upton, Long Island, NY 11973

70. Y. Sawada, Manager of Advanced Engineering Group, Heavy Apparatus Engineering Laboratory, Tokyo Shibaura Electric Co., Ltd. 4, 2-Chome, Suehiro-cho, Tsurumi-ku, Yokohama, Japan

71. K. Schmitter, Max-Planck-Institut für Plasmaphysik, 8046 Garching bei München, Federal Republic of Germany

72. J. Schultz, Massachusetts Institute of Technology, 167 Albany Street, Cambridge, MA 021.39

73. S. Shimamoto, Japan Atomic Energy Research Institute, Tokai Research Establishment, Tokai-mura, Naka-gun, Ibaraki-ken, Japan

74. M. Spadoni, Laboratorio Nazionali, Cas. Postale 70, 00044 Frascati, Italy

75. S. St. Lorant, Stanford Linear Accelerator Center, Sand Hill Road, Palo Alto, CA 94304

76. L. D. Stewart, Princeton University, Plasma Physics Laboratory, P.0. Box 451, Princeton, NJ 08540

77. B. P. Strauss, Magnetic Corporation of America, 179 Bear Hill Road, Palo Alto, CA 94304

78. K. Tachikawa, Chief of Electric Materials Laboratory, National Research Institute for Metals, 3-12, 2-Chome, Nakameguro, Meguru-ku, Tokyo, Japan

79. D. T. Uchida, Nuclear Engineering Department, University of Tokyo, Tokyo, Japan

80. S. T. Wang, Argonne National Laboratory, 9700 South Cass Avenue, Argonne, IL 60439

81. C. Waters, Technology Division, Building R25, Rutherford Laboratory, Chilton, Didcot, Oxfordshire, OX110QX, England

82. M. Wilson, Rutherford Laboratory, Chilton, Didcot, Oxfordshire, OX] lonX, F.ngl and

83. S. L. Wipf, Q-26, Los Alamos Scientific Laboratory, P.O. Box 1663, Los Alamos, NM 87544

84. H. H. Woodson, Chairman, Department of Electrical Engineering, University of Texas, Austin, TX 78712

85. M. Yamamoto, Toshiba, Tsurumi Works, 4, Suehiro-choizchome, Tsurumi-ku, Yokohama, 230, Japan

86. K. Yasukochi, Department of Physics, College of Scicnce and Engineering, Nihon University, Kanada-Surugadai, Chiyoda-ku, Tnykn, Tapan.

87. Office of Assistant Manager for Energy Research and Development, Department of Energy, Oak Ridge Operations Office, Oak Ridge, TN 37830

88-239. Given distribution as shown in TID-4500, Magnetic Fusion Energy (Distribution Category UC-20 b, Magnetic Systems) 